\title{
CORRECTION
}

View Article Online

View Journal I View Issue

\section{Correction: Inorganic p-type contact materials for perovskite-based solar cells}

Cite this: J. Mater. Chem. A, 2015, 3, 9318

\author{
Ming-Hsien Li, ${ }^{a}$ Po-Shen Shen, ${ }^{a}$ Kuo-Chin Wang, ${ }^{a}$ Tzung-Fang Guo abc \\ and Peter Chen*abc
}

DOI: $10.1039 / c 5 t a 90087 e$

www.rsc.org/MaterialsA

Correction for 'Inorganic p-type contact materials for perovskite-based solar cells' by Ming-Hsien Li et al., J.

Mater. Chem. A, 2015, DOI: 10.1039/c4ta06425a.

A relevant article (J. Mater. Chem. A, 2014, 2, 12754-12760) was not included in the above review however it is included in the revised Table 1 and Fig. 11 below.

Table 1 Summary of photovoltaic parameters of PSC based on inorganic p-type semiconductor

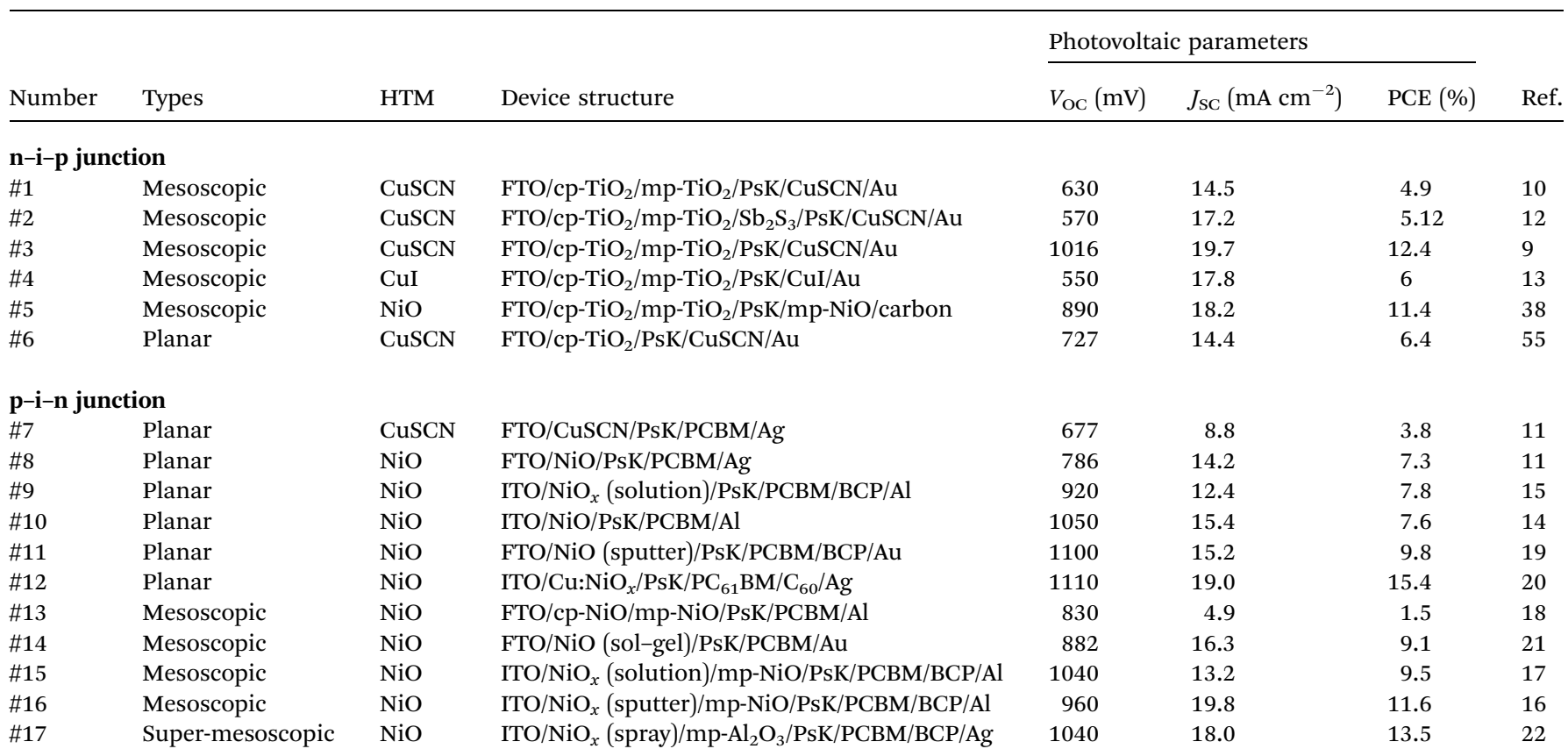

\footnotetext{
aDepartment of Photonics, National Cheng Kung University, Tainan, Taiwan 701. E-mail: petercyc@mail.ncku.edu.tw ${ }^{b}$ Research Center for Energy Technology and Strategy (RCETS), National Cheng Kung University, Tainan, Taiwan 701 'Advanced Optoelectronic Technology Center (AOTC), National Cheng Kung University, Tainan, Taiwan 701
} 


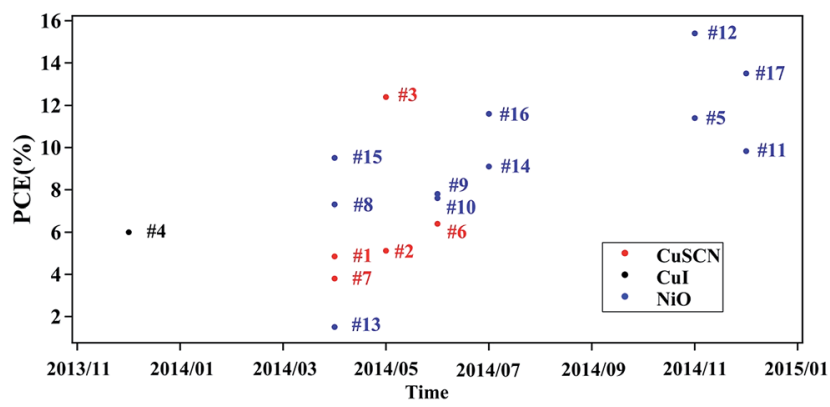

Fig. 11 PCE revolution of PSC based on inorganic $p$-type semiconductor.

\section{Reference}

55 S. Chavhan, O. Miguel, H.-J. Grande, V. Gonzalez-Pedro, R. S. Sánchez, E. M. Barea, I. Mora-Seró and R. Tena-Zaera, J. Mater. Chem. A, 2014, 2, 12754-12760.

The Royal Society of Chemistry apologises for these errors and any consequent inconvenience to authors and readers. 